\title{
Photosensitization, a Green Treatment for the Inactivation of Aspergillus Flavus in Peanuts Mediated by Curcumin ${ }^{+}$
}

\author{
Nalukui Mukubesa ${ }^{1, *}$, Hung T. Hong ${ }^{2}$, Maral Seidi Damyeh ${ }^{2}$ and Yasmina Sultanbawa ${ }^{2}$ \\ 1 School of Agriculture and Food Sciences, The University of Queensland, St Lucia QLD 4072, Australia \\ 2 Queensland Alliance for Agriculture and Food Innovation, Brisbane 4108, Australia; \\ h.trieu@uq.edu.au (H.T.H.); s.maral@uq.edu.au (M.S.D.); y.sultanbawa@uq.edu.au (Y.S.) \\ * Correspondence: nalukuimukubesa@gmail.com \\ + Presented at the third International Tropical Agriculture Conference (TROPAG 2019), Brisbane, Australia, \\ 11-13 November 2019.
}

Published: 16 March 2020

\begin{abstract}
The aim of this study was to investigate the efficiency of curcumin-based photosensitization (CUR-PS) in inactivating Aspergillus flavus spores and in turn reducing the aflatoxin B1 production and extending the shelf-life of raw peanuts. The inoculated peanuts with $A$. flavus spores were illuminated for $15 \mathrm{~min}$ at $420 \mathrm{~nm}$ (Xenon Arc light source) using three different curcumin concentrations of 50,75, and $100 \mu \mathrm{M}$. The highest reduction in A. flavus spores on peanuts (1.7 $\log \mathrm{CFU})$ was obtained by $50 \mu \mathrm{M}$ curcumin $(p>0.05)$. Interestingly, shelf life of treated peanuts with $75 \mu \mathrm{M}$ curcumin was extended by 7 days. In order to evaluate the influence of CUR-PS treatment on aflatoxin B1 generation, treated and untreated peanuts were analysed after 20 days of storage $25{ }^{\circ} \mathrm{C}$. Results showed that the average level of aflatoxin B1 in untreated (control) peanuts was $800 \mathrm{mg} / \mathrm{kg}$, while light treatment of peanuts reduced the aflatoxin production to the level of 296 $\mathrm{mg} / \mathrm{kg}$. A significant reduction in aflatoxin production was observed in curcumin $(75 \mu \mathrm{M})$ treated peanuts, where 38.3 and $41.1 \mathrm{mg} / \mathrm{kg}$ aflatoxin were detected in illuminated and not-illuminated peanuts, respectively. Moreover, 59.2 and $58.1 \mathrm{mg} / \mathrm{kg}$ aflatoxin were detected in CUR-PS treated peanuts with 50 and $100 \mu \mathrm{M}$ curcumin, respectively. However, not illuminating curcumin treated peanuts with 50 and $100 \mu \mathrm{M}$ showed higher levels of aflatoxin, 108.8 and $105.3 \mathrm{mg} / \mathrm{kg}$, respectively $(p>0.05)$. This study demonstrated the efficiency of CUR-PS treatment as a safe and green technology to control fungal growth and mycotoxin production in agro-food sector.
\end{abstract}

Keywords: photosensitization; curcumin; aflatoxin B1; peanuts; Aspergillus flavus

Author Contributions: The experimental design and procedures, objectives and data analysis were conducted by myself as a Masters student under the guidance and supervision of A/Professor Y.S. and the assistance of M.S.D. The design of the experiment, the scope and direction of the whole experiment came about through discussions with my supervisor, Y.S., and PhD student M.S.D. who also assisted in supervising the laboratory experiments. The experimental design and analysis of the aflatoxin levels in peanuts was mainly done by Dr H.T.H.

Funding: This project was jointly supported by QAAFI (Queensland Alliance for Agriculture and Food Innovation) and the School of Agriculture and Food Sciences (SAFS) under The University of Queensland.

Acknowledgments: I would like to thank the staff of QAAFI and DAF for their technical support as the experiment was conducted on their premises. I would also like to thank the teaching staff from SAFS for the guidance that they rendered including the administrative support provided. My sincere thanks go to the Australia Awards for sponsoring the whole Master's program. 
Conflicts of Interest: The authors declare no conflict of interest. 